\title{
On Chebsyshev Solution of Curves by Using Gaussian Curvature
}

\author{
Sibel Paşalı Atmaca* \\ Muğla Sitkı Koçman University, \\ 05536243638, sibela@mu.edu.tr \\ Received: 21 December 2016 \\ Accepted: 29 May 2017 \\ DOI: $10.18466 /$ cbayarfbe.339350
}

Gaussian curvature is commonly seen in the study of differential geometry. Gaussian curvature of a surface at a point is the product of the principal curvatures. They measure how the surface bends by different amounts in different directions at the point. Also, Gaussian curvature is given as the determinant of shape operator. In pure mathematics, differential equations are studied from different viewpoints. There are a lot of methods for solving differential equations in mathematics. From the differential equations viewpoint, Gaussian curvature solves the differential equation to find the main curve. One of them is Chebsyshev expansion method by using Chebsyshev polynomials. Also, they are important study in approximation theory. Chebyshev polynomials are a sequence of orthogonal polynomials and compose a polynomial sequence.The series solution is also used in surface of revolution. A surface of revolution is a surface generated by rotating a two-dimensional curve. In this study, our aim is to find the main curve by using Gaussian curvature. We substitute solution into the differential equation to find a relation for coeeficients of system. So, we use Chebsyshev polynomials for solutions to determine the curve and demonstrate our results on some well-known surfaces such as sphere, catenoid and torus.

AnahtarKelimeler - Chebsyshev Polynomials, Gaussian Curvature, Differential Equations, Shape Operator, Surface of Revolution.

\section{Introduction}

In differential geometry, the Gaussian curvature $\kappa$ of a surface at a point is the product of the principal curvatures $k_{1}$ and $k_{2}$ :

$$
\kappa=k_{1} k_{2}
$$

At a point $p$ on surface in $I R^{3}$, the Gaussian curvature is also given by

$$
\kappa(p)=\operatorname{det} S(p)
$$

where $S$ is the shape operator $[2,3,4,6]$. Also, Gaussian curvature solves the differential equation to find the main curve.

In mathematics, there are a lot of methods for solving differential equations. One of them is Chebsyshev expansion method by using Chebsyshev polynomials. The Chebsyshev polynomials are polynomials of degree $n$ [1]. The Chebyshev polynomials of the first kind are defined by

$$
\begin{aligned}
& T_{0}(x)=1, \\
& T_{1}(x)=x,
\end{aligned}
$$

$$
T_{n+1}(x)=2 x T_{n}(x)-T_{n-1}(x) .
$$

A surface of revolution can be obtained by rotating a curve in the $x z$-plane about the $z$-axis. Suppose that the curve is given by

$$
\mathrm{x}=\varphi(t), \mathrm{z}=\Psi(t)
$$

with $t$ lies in $(a, b)$, and is parametrized by arclength, so that

$$
\dot{\varphi}^{2}+\dot{\Psi}^{2}=1
$$

The coefficients of first fundamental form are given by

$$
\begin{gathered}
E=\varphi^{2}, \\
F=0, \\
G=(\dot{\varphi})^{2}+(\dot{\Psi}) .
\end{gathered}
$$

The coefficients of second fundamental form are given by

$$
\begin{gathered}
e=\varphi \dot{\Psi}, \\
f=0, \\
g=\dot{\Psi} \ddot{\varphi}-\Psi \ddot{\varphi} .
\end{gathered}
$$

Then, we obtain

$$
\kappa(t)=\frac{e g-f^{2}}{E G-F^{2}}=\frac{\dot{\Psi}\left(\dot{\Psi} \ddot{\varphi}-\Psi_{\varphi}\right)}{\varphi}
$$

If we differentiate equation (1), we find

$$
\dot{\varphi} \ddot{\varphi}=-\dot{\Psi} \ddot{\Psi}(2)
$$

After some calculations, we get

$$
K(t)=-\frac{\ddot{\varphi}}{\varphi} .
$$


Celal Bayar University Journal of Science

Volume 13, Issue 3, p 745-746

Our aim is to find the curve $\varphi$ by using Chebsyshev polynomials. Then, we substitute solution into the differential equation to find relation for the coefficients. The results are applied on some well-known surfaces such as sphere, catenoid and torus.

\section{Method}

$$
\ddot{\varphi}+K(x) \varphi=0
$$

If we substitute $\varphi=\sum_{n=0}^{\infty} A_{n} T_{n}(x), \dot{\varphi}=$

$\sum_{n=1}^{\infty} A_{n}\left(T_{n}(x)\right)^{\prime}$ and $\ddot{\varphi}=\sum_{n=2}^{\infty} A_{n}\left(T_{n}(x)\right)^{\prime \prime}$ into equation (4), we obtain

$$
\sum_{n=2}^{\infty} A_{n}\left(T_{n}(x)\right)^{\prime \prime}+K(x) \sum_{n=0}^{\infty} A_{n} T_{n}(x)=0
$$

After some calculations [7,8,9], we obtain

$$
\begin{gathered}
A_{2}=\frac{-\kappa(x) A_{0}}{4}, \\
A_{3}=\frac{-\kappa(x) A_{1}}{24}, \\
A_{4}=\frac{\left(2 x^{2}-1\right) \kappa^{2}(x) A_{0}}{4\left(96 x^{2}-16\right)},
\end{gathered}
$$

Hence, we can find the coefficients of $\varphi$.

$$
\begin{gathered}
\varphi=A_{0} T_{0}(x)+A_{1} T_{1}(x)+A_{2} T_{2}(x)+A_{3} T_{3}(x)+ \\
A_{4} T_{4}(x)+\cdots .
\end{gathered}
$$

Finally, we obtain

$$
\begin{array}{r}
\varphi=\frac{1}{\pi} \int_{-1}^{1} \frac{\varphi(x) d x}{\sqrt{1-x^{2}}}\left(1-\frac{\kappa(x)}{4}+\frac{\left(2 x^{2}-1\right) \kappa^{2}(x)}{4\left(96 x^{2}-16\right)}+\cdots\right)+ \\
\quad \frac{2}{\pi} \int_{-1}^{1} \frac{x \cdot \varphi(x) d x}{\sqrt{1-x^{2}}}\left(x-\frac{\kappa(x)}{24}+\frac{\left(4 x^{2}-3\right) \kappa^{2}(x)}{320 x^{2}-120}-\cdots\right) .(7)
\end{array}
$$

\section{Examples}

The Gaussian curvature of paraboloid which has radius $a$ at height $h$ is

$$
K(x)=\frac{4 h^{2}}{\left(a^{2}+4 h x\right)} \cdot(8)
$$

We can find that

$$
\begin{gathered}
\varphi=\frac{1}{\pi} \int_{-1}^{1} \frac{\varphi(x) d x}{\sqrt{1-x^{2}}} \quad\left(1-\frac{h^{2}}{\left(a^{2}+4 h x\right)}+\frac{\left(2 x^{2}-1\right) 4 h^{2}}{\left(a^{2}+4 h x\right)\left(96 x^{2}-16\right)}+\right. \\
\cdots)+\frac{2}{\pi} \int_{-1}^{1} \frac{x \cdot \varphi(x) d x}{\sqrt{1-x^{2}}}\left(x-\frac{h^{2}}{6\left(a^{2}+4 h x\right)}+\right. \\
\left.\frac{\left(4 x^{2}-3\right) 16 h^{2}}{\left(320 x^{2}-120\right)\left(a^{2}+4 h x\right)}-\cdots\right) .
\end{gathered}
$$

The Gaussian curvature of torus which has the radius from the center of the hole to the center of the torus tube and the radius of the tube be $a$ is

$$
K(x)=\frac{\cos x}{a(c+a \cos x)} .
$$

We can find that

$$
\begin{aligned}
& \varphi=\frac{1}{\pi} \int_{-1}^{1} \frac{\varphi(x) d x}{\sqrt{1-x^{2}}}\left(1-\frac{\cos x}{4 a(c+a \cos x)}+\right. \\
& \left.\frac{\left(2 x^{2}-1\right) \cos ^{2} x}{4 a^{2}(c+a \cos x)^{2}\left(96 x^{2}-16\right)}+\cdots\right)+\frac{2}{\pi} \int_{-1}^{1} \frac{x \cdot \varphi(x) d x}{\sqrt{1-x^{2}}}(x- \\
& \left.\frac{\cos x}{24 a(c+a \cos x)}+\frac{\left(4 x^{2}-3\right) \cos ^{2} x}{\left(320 x^{2}-120\right)(c+a \cos x)^{2}}-\cdots\right)
\end{aligned}
$$

The Gaussian curvature of catenoid is [5]

$$
K(x)=-\frac{1}{c^{2}} \sec ^{4} \frac{x}{c}
$$

We can find that

$$
\begin{aligned}
& \varphi=\frac{1}{\pi} \int_{-1}^{1} \frac{\varphi(x) d x}{\sqrt{1-x^{2}}}\left(1+\frac{\sec ^{4} \frac{x}{c}}{4 c^{2}}+\frac{\left(2 x^{2}-1\right) \sec ^{8} \frac{x}{c}}{4\left(96 x^{2}-16\right) c^{4}}+\cdots\right)+ \\
& \frac{2}{\pi} \int_{-1}^{1} \frac{x \cdot \varphi(x) d x}{\sqrt{1-x^{2}}}\left(x+\frac{\sec ^{4} \frac{x}{c}}{24 c^{2}}+\frac{\left(4 x^{2}-3\right) \sec ^{8} \frac{x}{c}}{\left(320 x^{2}-120\right) c^{4}}-\cdots\right) .
\end{aligned}
$$

\section{References}

1. Adomian, G., Convergent series solution of nonlinear equations, Journal of Computational and Applied Mathematics, 1984, 11(1), 225-230.

2. Carmo, M. P. DiferansiyelGeometri: EğrilerveYüzeyler, Ankara, 2012.

3. Meek, D. S., Walton, D. J., On surface normal and Gaussian curvature approximations given data sampled from a smooth surface, Computer Aided Geometric Design, 2000, 17(6), 521-543.

4. Han, Z., Prescribing Gaussian curvature on S2, Duke Mathematical Journal, 1990, 61(3), 679.

5. Stewart, J., Calculus, Fourth edition, United States, 1999.

6. Hacısalihoglu , H. H., Diferansiyel Geometri, Ankara,1998.

7. Schoen, R., Zhang, D., Prescribed scalar curvature on the n-sphere, Calculus of Variations and Partial Differential Equations, 1996, 4(1), 125.

8. Hua Hau, Z., Hypersurfacesin a sphere with constant mean curvature, Proceedings of the American Mathematical Society, 1997, 125, 11931196.

9. Carmo, M., Dajczer, M., Rotation hypersurfaces in spaces of constant curvature, Transactions of the American Mathematical Society, 1983, 277, 685-709. 